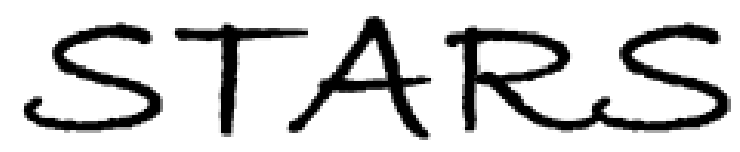

University of Central Florida

STARS

Faculty Bibliography 2000s

Faculty Bibliography

$1-1-2004$

\title{
Dual-frequency liquid crystal gels with submillisecond response time
}

\author{
Yun-Hsing Fan \\ University of Central Florida \\ Hongwen Ren \\ University of Central Florida \\ Xiao Liang \\ University of Central Florida \\ Yi-Hsin Lin \\ University of Central Florida \\ Shin-Tson Wu \\ University of Central Florida
}

Find similar works at: https://stars.library.ucf.edu/facultybib2000

University of Central Florida Libraries http://library.ucf.edu

This Article is brought to you for free and open access by the Faculty Bibliography at STARS. It has been accepted for inclusion in Faculty Bibliography 2000s by an authorized administrator of STARS. For more information, please contactSTARS@ucf.edu.

\section{Recommended Citation}

Fan, Yun-Hsing; Ren, Hongwen; Liang, Xiao; Lin, Yi-Hsin; and Wu, Shin-Tson, "Dual-frequency liquid crystal gels with submillisecond response time" (2004). Faculty Bibliography 2000s. 4344.

https://stars.library.ucf.edu/facultybib2000/4344

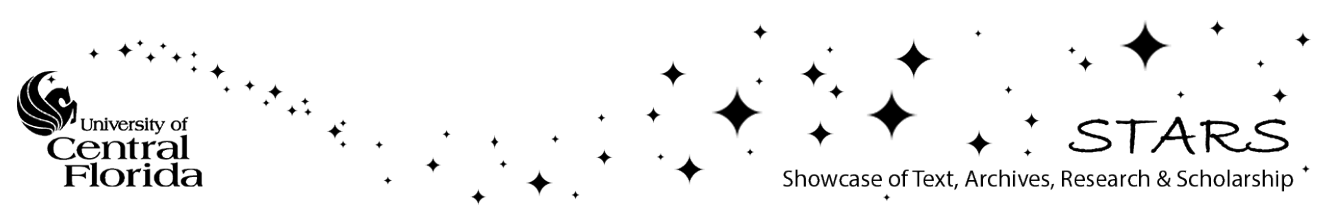




\section{Dual-frequency liquid crystal gels with submillisecond response time}

Cite as: Appl. Phys. Lett. 85, 2451 (2004); https://doi.org/10.1063/1.1799249

Submitted: 25 May 2004 . Accepted: 27 July 2004 . Published Online: 28 September 2004

Yun-Hsing Fan, Hongwen Ren, Xiao Liang, Yi-Hsin Lin, and Shin-Tson Wu

\section{ARTICLES YOU MAY BE INTERESTED IN}

Fast switching dual-frequency liquid crystal optical retarder, driven by an amplitude and frequency modulated voltage

Applied Physics Letters 83, 3864 (2003); https://doi.org/10.1063/1.1625114

Dielectric heating effects of dual-frequency liquid crystals

Applied Physics Letters 86, 231104 (2005); https://doi.org/10.1063/1.1944889

Dual-frequency addressed hybrid-aligned nematic liquid crystal

Applied Physics Letters 85, 3354 (2004); https://doi.org/10.1063/1.1809282

\section{Applied Physics Letters}

Mid-IR and THz frequency combs special collection

Read Now! 


\title{
Dual-frequency liquid crystal gels with submillisecond response time
}

\author{
Yun-Hsing Fan, Hongwen Ren, Xiao Liang, Yi-Hsin Lin, and Shin-Tson Wu ${ }^{\text {a) }}$ \\ College of Optics and Photonics, University of Central Florida, Orlando, Florida 32816
}

(Received 25 May 2004; accepted 27 July 2004)

\begin{abstract}
Two types of gels using dual-frequency liquid crystal are demonstrated. The one using a homogeneous cell shows anisotropic scattering behavior while the other prepared using a cell without polyimide alignment layers exhibits isotropic scattering properties. Both liquid crystal gels are highly transparent in the voltage-off state. Light scattering occurs when a high frequency voltage is applied. The isotropic gel exhibits a high contrast ratio and submillisecond response time. Potential applications of these gels for switchable polarizer, telecom optical switch, and reflective displays are emphasized. (c) 2004 American Institute of Physics. [DOI: 10.1063/1.1799249]
\end{abstract}

Liquid crystal (LC) gel (also known as polymerstabilized LC) which normally consists of 3-8 wt \% polymer in a nematic host exhibits an anisotropic light scattering behavior and has been used as switchable polarizer, light shutter, and reflective display. ${ }^{1-7}$ By reducing the domain size, light scattering could be eliminated in the near infrared spectral region. Such a LC polymer network exhibits a fast response time except for the increased operating voltage. ${ }^{8}$

Both reverse-mode and normal-mode LC gels have been developed. ${ }^{5,9}$ The reverse mode gel is highly transparent in the voltage-off state. As the voltage exceeds a threshold, the gel is switched into a multidomain structure and the incident light polarized in the rubbing direction is scattered. For a homogeneous alignment gel using a positive dielectric anisotropy $(\Delta \varepsilon>0)$ LC, the contrast ratio could exceed 2000:1, depending on the curing conditions. ${ }^{6}$ However, the device is polarization sensitive. For a homeotropic alignment gel employing a negative $\Delta \varepsilon$ LC, the contrast ratio is only $\sim 10: 1$ for an unpolarized light. ${ }^{2}$ The response time of both gels is $\sim 10 \mathrm{~ms}$, depending on the LC material employed and polymer concentration.

In this letter, we demonstrate fast response time and high contrast ratio gels using a dual-frequency liquid crystal (DFLC). ${ }^{10}$ For a homogeneous gel, the light scattering is anisotropic. However, for a DFLC gel prepared using substrates without alignment layer, the light scattering is independent of polarization and the response time is $\sim 0.8 \mathrm{~ms}$.

The LC gels were fabricated by photopolymerizing the DFLC/monomer mixture. The DFLC mixture we employed consists of some biphenyl esters and lateral difluoro tolanes. Its physical properties are summarized as follows: cross-over frequency $f_{c} \sim 5 \mathrm{kHz}$, birefringence $\Delta n \sim 0.277$ (at $\lambda$ $=633 \mathrm{~nm}$ and $T=22{ }^{\circ} \mathrm{C}$ ), and dielectric anisotropy $\Delta \varepsilon=6.6$ at $f=1 \mathrm{kHz}$ and $\Delta \varepsilon=-4.1$ at $50 \mathrm{kHz}$. Two samples with 5 and $7 \mathrm{wt} \%$ monomer concentration were prepared. The photocurable monomer used in this study is bisphenol A dimethacrylate. ${ }^{9}$ The LC/monomer mixture was filled in the cells with gap $d=8 \mu \mathrm{m}$. In our experiment, two types of LC cells were studied: one has homogeneous alignment and the other has no polyimide alignment layer. For the homogeneous cell, the inner surfaces of the indium-tin-oxide glass substrates were overcoated with a thin polyimide layer and buffed in antiparallel directions. In both cells, the LC/

\footnotetext{
${ }^{a)}$ Electronic mail: swu@mail.ucf.edu
}

monomer mixture was irradiated by an UV light $(\lambda$ $\sim 365 \mathrm{~nm})$ under the application of a bias voltage $\left(V_{b}\right.$ $\left.=15 V_{\text {rms }}\right)$ across the cell so that the LC and monomer molecules were oriented at nearly perpendicular to the substrates. As a result, the formed chain-like polymer networks are along the electric field direction. The UV curing intensity was $I \sim 20 \mathrm{~mW} / \mathrm{cm}^{2}$ and curing time $\sim 10 \mathrm{~min}$.

We measured the transmittance of both LC gels using a $\mathrm{He}-\mathrm{Ne}$ laser beam at normal incidence angle. The laser beam diameter is $\sim 1 \mathrm{~mm}$. A photodiode detector was set at $\sim 25 \mathrm{~cm}$ away from the LC sample, which corresponds to $\pm 1^{\circ}$ acceptance angle. A linearly polarized $\mathrm{He}-\mathrm{Ne}$ laser was used for studying the anisotropic scattering behavior of the homogeneous gel and an unpolarized $\mathrm{He}-\mathrm{Ne}$ laser was used to characterize the isotropic DFLC gel.

Figure 1 shows the voltage-dependent transmittance of the $8 \mu \mathrm{m}$ homogeneous LC gels at two monomer concentrations $(c), c=5$ and $7 \mathrm{wt} \%$. The $100 \%$ transmittance in Fig. 1 represents the laser transmission of an $8 \mu \mathrm{m}$ homogeneous cell filled with the DFLC mixture alone. In the gel experiment, the laser polarization is parallel to the LC rubbing direction. In the voltage-off state, the polymer networks align LC molecules perpendicular to the substrates, similar to a homeotropic LC cell shown in Fig. 2(a). When a lowfrequency $(f=1 \mathrm{kHz})$ electric field is applied to the cell, the LC molecules are aligned more orderly (perpendicular to the substrates). As a result, the transmission is slightly increased,

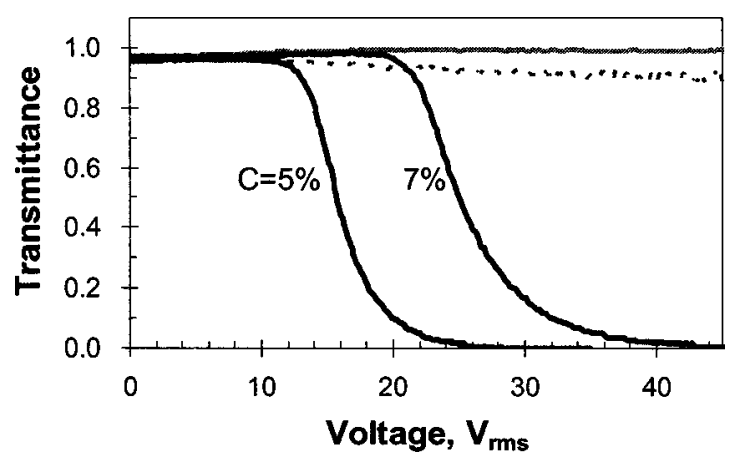

FIG. 1. Voltage-dependent normalized transmittance of the homogeneousaligned DFLC gels at different monomer concentrations. Solid and gray lines are for $f=50$ and $1 \mathrm{kHz}$, respectively, and polarization $\|$ to the cell rubbing direction. Dashed lines are for $c=5 \mathrm{wt} \%$ and $f=50 \mathrm{kHz}$, but polarization $\perp$ to the cell rubbing direction. Cell gap $d=8 \mu \mathrm{m}, \lambda=633 \mathrm{~nm}$, and $T=22{ }^{\circ} \mathrm{C}$. 


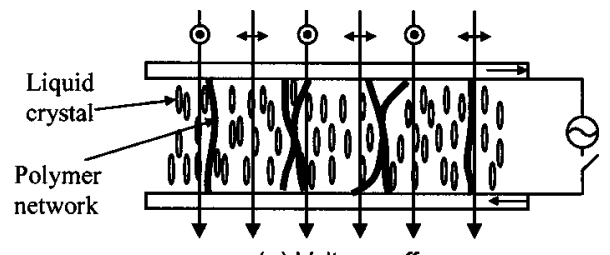

(a) Voltage-off

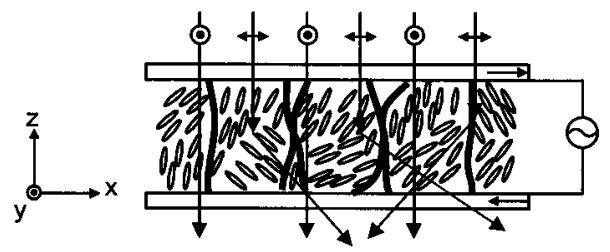

(b) Voltage-on $(50 \mathrm{kHz})$

FIG. 2. Schematic representation of the operating principle of homogeneous-aligned DFLC gels. (a) Voltage-off state and (b) voltage-on state. The arrows in the substrates indicate the rubbing directions.

as shown by the gray line in Fig. 1. If a high frequency $(f$ $=50 \mathrm{kHz})$ voltage is applied, the DFLC exhibits a negative dielectric anisotropy. The cell is equivalent to a negative LC in a homeotropic cell. As the applied voltage exceeds a threshold $\left(\sim 12 V_{\mathrm{rms}}\right.$ for the $\left.c=5 \mathrm{wt} \% \mathrm{gel}\right)$, the LC molecules are switched into a multi-domain structure, as illustrated in Fig. 2(b). Thus, light scattering takes place and transmittance begins to decrease. The LC directors in different domains have different orientations. The resultant refractive index mismatch scatters light at the interfaces between domains. Due to the antiparallel alignment layers on the substrates, the LC molecules will be reoriented in the $x-z$ plane. For the light with polarization perpendicular to the rubbing direction, it encounters the ordinary refractive index $n_{o}$ in both voltage-off and -on states. This ordinary ray passes through the cell without being scattered. On the other hand, the light polarized along the rubbing direction is scattered. The measured contrast ratio is $\sim 360: 1$ for the $c=5 \mathrm{wt} \%$ sample.

To measure the response time of the DFLC gel, we could switch voltage at a high frequency or switch frequency at a fixed voltage. In the case of switching voltage between 0 and $30 V_{\mathrm{rms}}(f=50 \mathrm{kHz})$, the rise time was measured to be $2 \mathrm{~ms}$, and decay time $21 \mathrm{~ms}$. The faster rise time is due to the voltage assisted molecular reorientation. ${ }^{11}$ If we fix the voltage at $30 V_{\text {rms }}$ while switching frequency between 1 and $50 \mathrm{kHz}$, the rise and decay time is reduced to 2 and $5.5 \mathrm{~ms}$, respectively. At the translucent state, the gel scatters the light polarized along the buffing direction so that the transmitted light remains linearly polarized. Such an anisotropic LC gel can be used as a white light switchable polarizer or reflective display. ${ }^{5}$

The polymer concentration plays a very important role affecting the contrast ratio and gel stability. A too small monomer concentration results in lower density polymer networks and weaker polymer anchoring force, which makes the polymer network unable to hold the LC molecules perpendicular to the substrates. In our experiment, we found if the monomer concentration is below $5 \mathrm{wt} \%$, the gel becomes irreversible after having been switched for several cycles. On the other hand, a high monomer concentration leads to an increased threshold voltage. From Fig. 1, the threshold voltage of the $7 \% \mathrm{LC}$ gel is $\sim 20 \mathrm{~V}_{\text {rms. }}$.

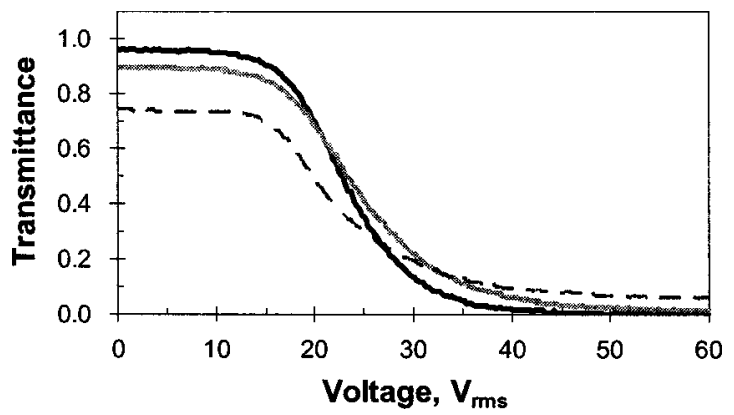

FIG. 3. Voltage-dependent normalized transmittance of the DFLC gels with monomer concentration of $5 \mathrm{wt} \%$ at $15 V_{\text {rms }}$ (solid lines), $8 V_{\text {rms }}$ (gray lines), and $4 V_{\text {rms }}$ (dashed lines) bias voltage during UV illumination.

The bias voltage during the curing process also affects the performance of the DFLC gels. Figure 3 plots the voltage-dependent transmittance of the $8 \mu \mathrm{m}$ DFLC gels at 4,8 , and $15 V_{\text {rms }}$ bias voltage. When the bias voltage is sufficiently higher than the threshold of the pure LC, most of the bulk LC molecules are reoriented perpendicular to the substrates. The resultant polymer networks are in a more ordered structure. If the bias voltage is too low, the cured LC molecules have a tilt angle with respect to the substrates. Its bright and dark states are not as good as those with high bias voltage, as depicted in Fig. 3.

To fabricate a polarization-independent DFLC gel, we inject the LC/monomer mixture into a cell without polyimide alignment layer. Figure 4 plots the voltage-dependent transmittance of such a DFLC gel. The monomer concentration is $5 \mathrm{wt} \%$ and cell gap $\mathrm{d}=8 \mu \mathrm{m}$. At $V=0$, the DFLC gel is in the uniform homeotropic state, similar to that shown in Fig. 2(a). The incident light propagates through the cell without scattering. When we apply an electric field with $f=50 \mathrm{kHz}$, the LC molecules are tilted away from the field direction. The LC/polymer composite is switched to a multidomain structure. The gel becomes optically nonuniform and therefore scatters the incident light. Since there is no preferred tilt direction, the LC directors in the polymer network domains are randomly distributed in the $x-y$ plane, as shown in Fig. 5. The gel is polarization-independent and can be used as a broadband light shutter. For the $c=5 \mathrm{wt} \%$ gel with $d$ $=8 \mu \mathrm{m}$, the contrast ratio was measured to be $\sim 160: 1$ for the unpolarized $\mathrm{He}-\mathrm{Ne}$ laser beam. A better dark state can be achieved at a higher voltage.

The response time of such an isotropic DFLC gel was measured. If we switch the applied voltage $(f=50 \mathrm{kHz})$ from 0 to $50 V_{\mathrm{rms}}$, the rise time is $0.8 \mathrm{~ms}$ and decay time is $10 \mathrm{~ms}$ for the $c=5 \mathrm{wt} \%$ sample. The observed response time

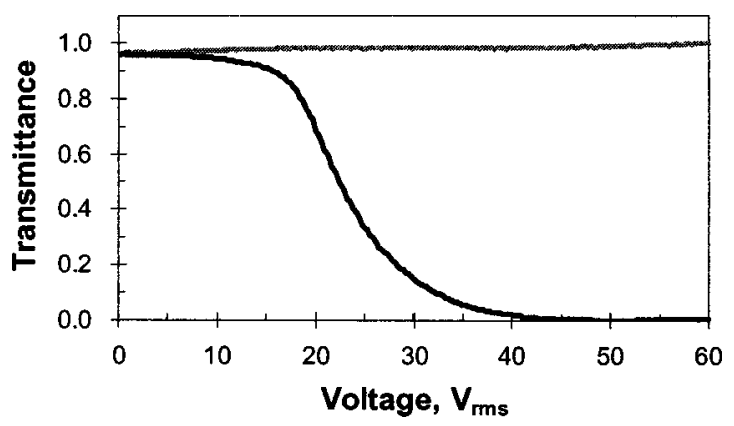

FIG. 4. Voltage-dependent normalized transmittance of the no-alignment DFLC gels with monomer concentration of $5 \mathrm{wt} \%$ in an $8 \mu \mathrm{m}$ cell. 


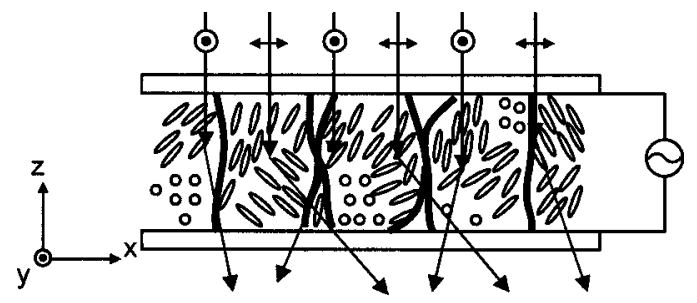

FIG. 5. Schematic representation of the operating principle of no-alignment DFLC gels in a voltage-on state. The frequency of applied voltage is $50 \mathrm{kHz}$.

of the isotropic gel is about $2 \times$ faster than the corresponding homogeneous DFLC gel. In the homogeneous cell, the buffed polyimide layers tend to align LC molecules parallel to the rubbing direction, but the polymer networks draw the LC directors perpendicular to the substrate. These two competing torques lead to a slower relaxation time. When we switch the isotropic gel at $50 V_{\text {rms }}$ between $f=1$ and $50 \mathrm{kHz}$, the rise time and decay time is each $0.8 \mathrm{~ms}$. Without polyimide layer, the fabrication process of the isotropic gel is much simpler.

Figure 6 shows the measured transmission spectra of the $8 \mu \mathrm{m}$ isotropic DFLC gel at voltage-on and -off states. The bright state was measured at $V=50 V_{\text {rms }}$ and $f=1 \mathrm{kHz}$ while the dark state was measured at the same voltage except for $f=50 \mathrm{kHz}$. Recall that the crossover frequency is $f_{c}=5 \mathrm{kHz}$. The spectral bandwidth covers the whole visible region.

In conclusion, we have demonstrated an anisotropic gel and an isotropic gel using a dual-frequency LC. The light scattering behavior for the homogeneous LC gel is dependent on the incident light polarization. However, the gel without alignment layer is polarization independent. These

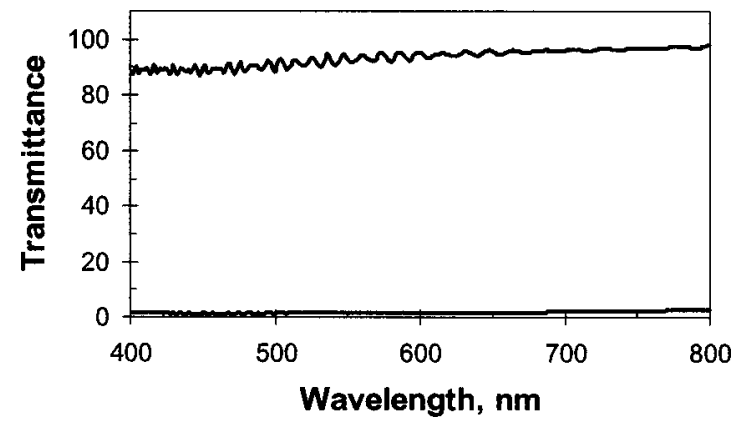

FIG. 6. The spectral bandwidth of an $8 \mu \mathrm{m}$ no-alignment DFLC gel. Upper trace: $V=50 V_{\text {rms }}$ at $f=1 \mathrm{kHz}$; lower trace: $V=50 V_{\text {rms }}$ at $f=50 \mathrm{kHz}$.

devices exhibit a high contrast ratio and fast response time. These LC gels can be used as a switchable polarizer and light shutter.

This work is supported by AFOSR under Contract No. F49620-01-1-0377.

${ }^{1}$ R. A. M. Hikmet and H. J. Boots, Phys. Rev. E 51, 5824 (1995).

${ }^{2}$ R. A. M. Hikmet, Liq. Cryst. 9, 405 (1991).

${ }^{3}$ R. A. M. Hikmet, Mol. Cryst. Liq. Cryst. 213, 117 (1992).

${ }^{4}$ R. Q. Ma and D. K. Yang, Phys. Rev. E 61, 1567 (2000).

${ }^{5}$ H. Ren and S. T. Wu, Appl. Phys. Lett. 82, 1432 (2002).

${ }^{6}$ F. Du and S. T. Wu, Appl. Phys. Lett. 83, 1310 (2003).

${ }^{7}$ R. L. Sutherland, V. P. Tondiglia, and L. V. Natarajan, Appl. Phys. Lett. 64, 1074 (1994).

${ }^{8}$ Y. H. Fan, Y. H. Lin, H. Ren, S. Gauza, and S. T. Wu, Appl. Phys. Lett. 84, 1233 (2004)

${ }^{9}$ Y. H. Fan, H. W. Ren, and S. T. Wu, Appl. Phys. Lett. 82, 2945 (2003).

${ }^{10}$ H. K. Bucher, R. T. Klingbiel, and J. P. VanMeter, Appl. Phys. Lett. 25, 186 (1974).

${ }^{11}$ S. T. Wu and D. K. Yang, Reflective Liquid Crystal Displays (Wiley, New York, 2001). 